\title{
Supporting Information \\ Wetting dynamics of a water droplet on micro-pillar surfaces with radially varying pitches
}

\author{
Manish Kumar ${ }^{1}$, Rajneesh Bhardwaj ${ }^{1}$, Kirti Chandra Sahu² ${ }^{*}$ \\ ${ }^{1}$ Department of Mechanical Engineering, Indian Institute of Technology Bombay, Mumbai 400076, India \\ ${ }^{2}$ Department of Chemical Engineering, Indian Institute of Technology Hyderabad, Sangareddy 502285 , \\ Telangana, India \\ ${ }^{*}$ Corresponding author (email: ksahu@iith.ac.in)
}

(a)

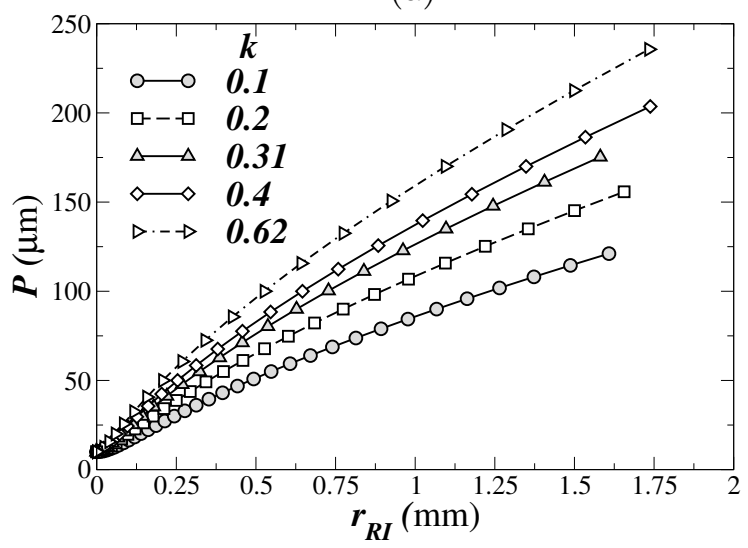

(b)

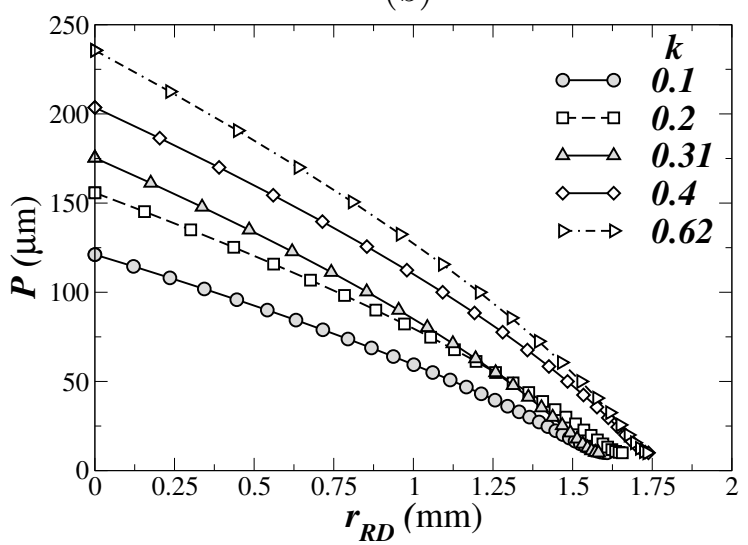

Figure S1: The variations of pitch $(P$ in $\mu \mathrm{m})$ with the radial distance from the center of the surface for different values of $k$ for the (a) radially increasing (RI) and (b) radially decreasing (RD) configurations for $S=10 \mu \mathrm{m}$. 

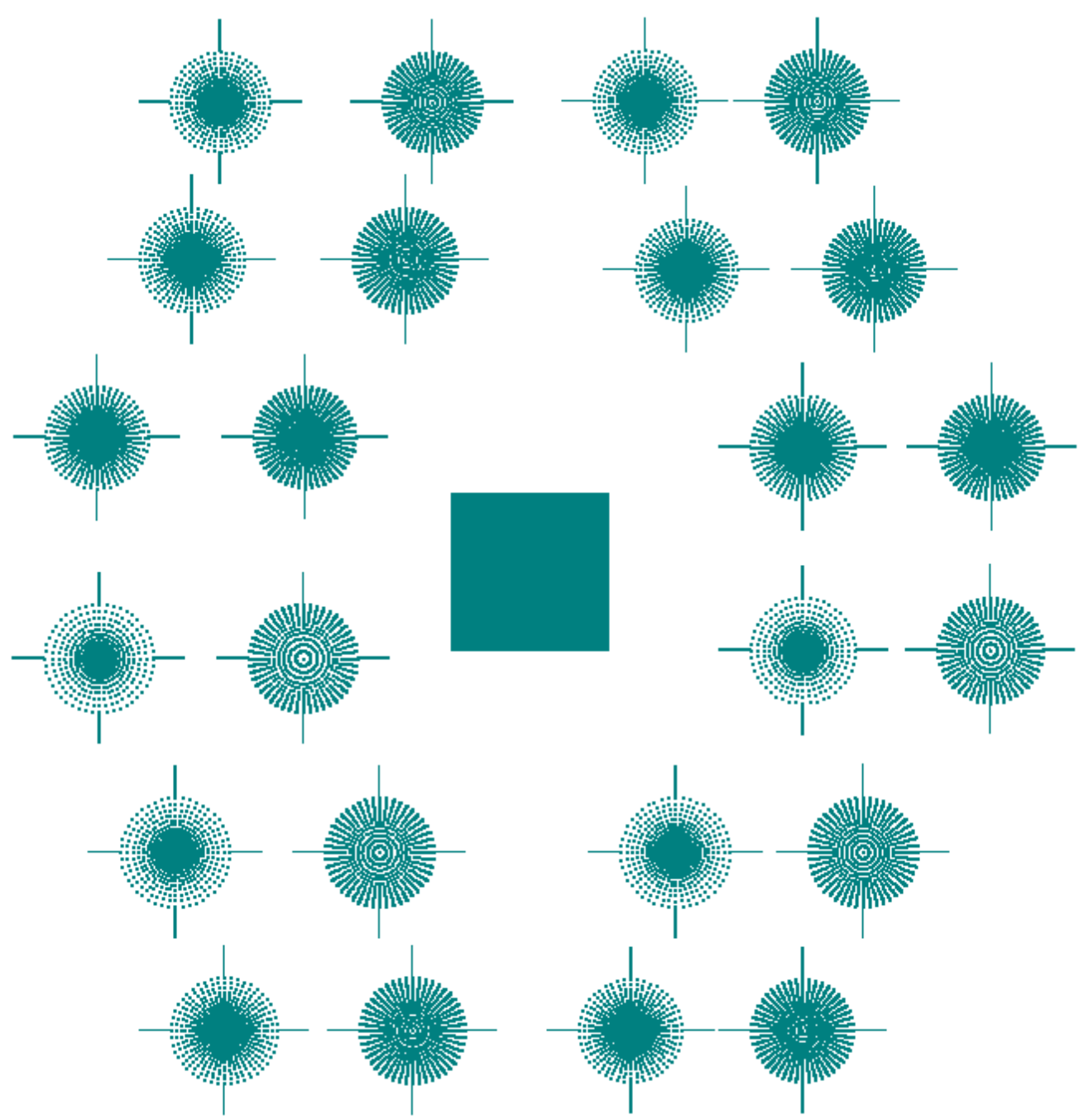

Figure S2: Snapshot of the CAD geometry showing the layout of fabricated SU-8 surfaces on single 2 inch silicon wafer. 

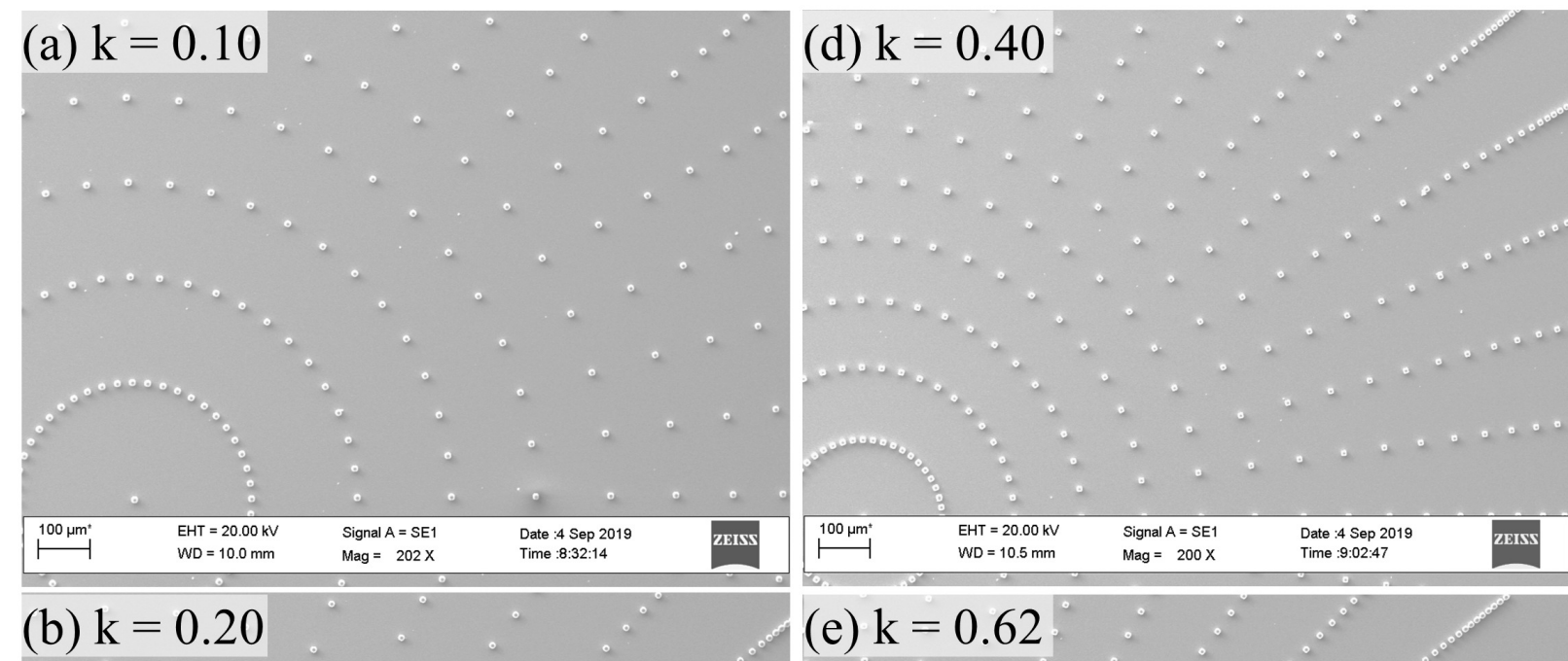

(e) $\mathrm{k}=0.62$
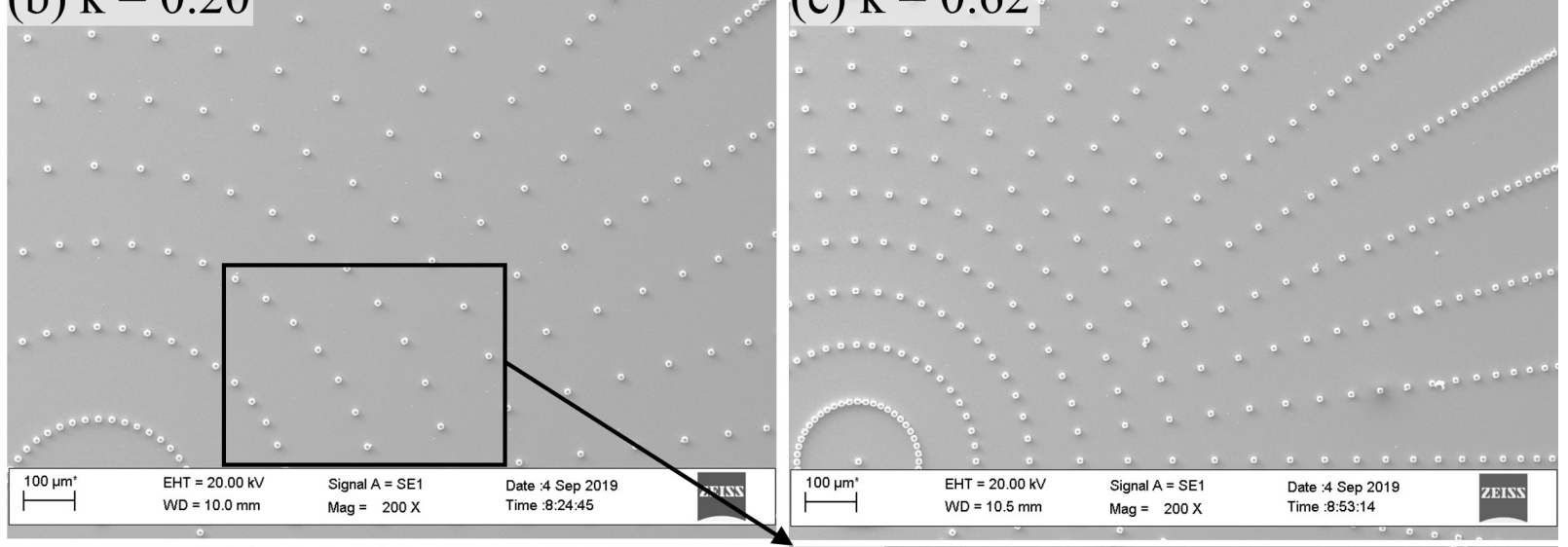

(c) $\mathrm{k}=0.31$

(b1)
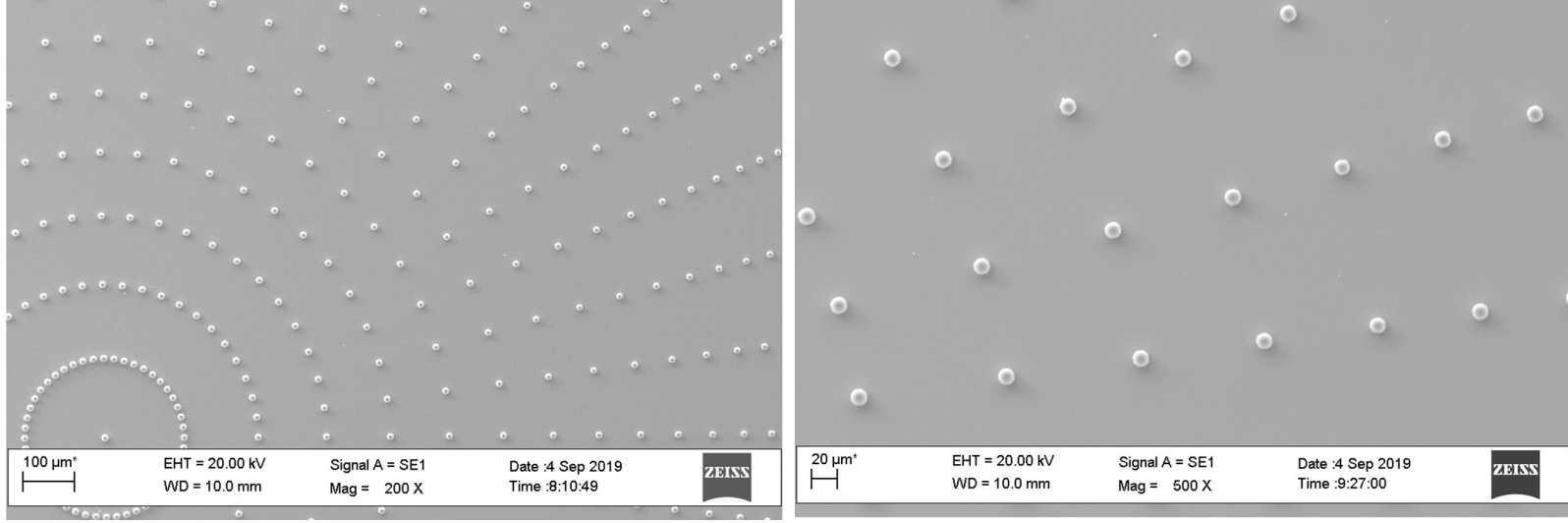

Figure S3: SEM images of the micro-pillar surfaces for different values of $k$ for the RI configuration having pillar size (S) $10 \mu \mathrm{m}$. Panel (b1) shows the zoomed in image of panel (b). 


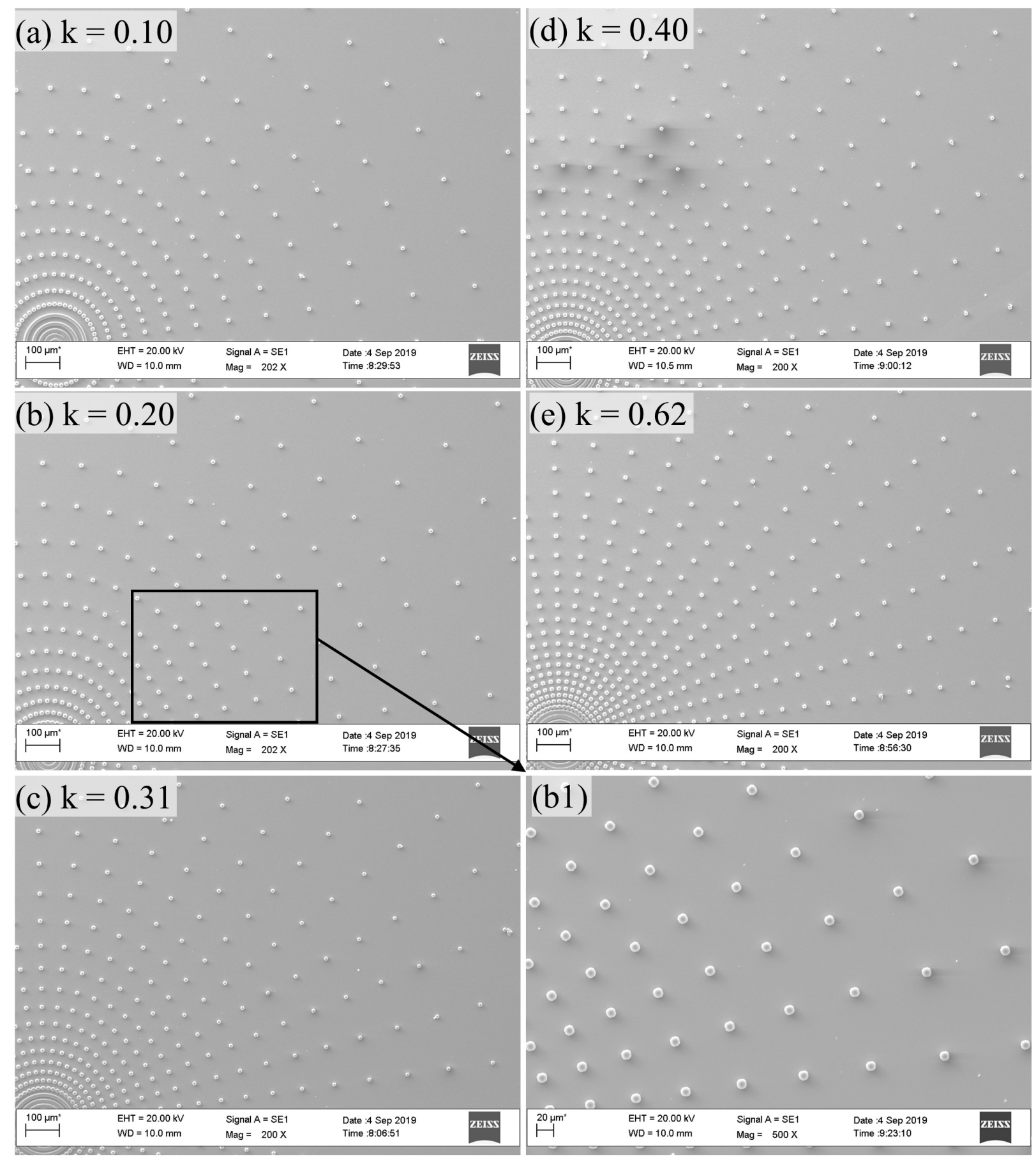

Figure S4: SEM images of the micro-pillar surfaces for different values of $k$ for the RD configuration having pillar size (S) $10 \mu \mathrm{m}$. 